\title{
An Efficient One-Pot Multi-Component Synthesis of 3,4-Dihydropyrimidin-2-(1H)-Ones/Thiones Catalyzed by Bismuth (III) Sulfate Trihydrate under Solvent-Free Conditions
}

\author{
Farhad Hatamjafari \\ Department of Chemistry, Faculty of Science, Islamic Azad University, Tonekabon Branch, Tonekabon, Iran \\ Correspondence should be addressed to Farhad Hatamjafari; hatamjafari@yahoo.com
}

Received 17 October 2013; Revised 8 January 2014; Accepted 8 January 2014; Published 13 March 2014

Academic Editor: Ashraf Aly Shehata

Copyright (C) 2014 Farhad Hatamjafari. This is an open access article distributed under the Creative Commons Attribution License, which permits unrestricted use, distribution, and reproduction in any medium, provided the original work is properly cited.

\begin{abstract}
A convenient and efficient protocol for the one-pot synthesis of 3,4-dihydropyrimidin-2-(1H)-one/thione derivatives of aldehydes, and 1,3-dicarbonyl compounds with Bismuth (III) sulfate trihydrate as the catalyst was described. We had the advantages of good performance, simplicity, and short time reaction under solvent-free conditions. The catalyst can be repeatedly reused without loss of its activity.
\end{abstract}

\section{Introduction}

Multi-component reactions play an important role in pharmaceutical industries. Pharmacies are trying to develop green chemistry reactions. Solvent-free synthesis of complex organic structures as drugs is the dream of every pharmacy. Multi-component reaction as a powerful tool for the rapid introduction of molecular diversity is evident and developed for the generation of heterocycles which receive growing interest [1-3]. Biginelli reaction is one of the most important multi-component reactions for the synthesis of dihydropyrimidinones/thiones. 3,4-Dihydropyrimidin-2 $(1 \mathrm{H})$-ones/thiones (DHPMs) reported the activity of many drugs as having antiviral, antibacterial, and antihypertensive effects, as calcium channel modulators [4-7], and as multidrug resistance reversal $[8,9]$. Biological activity of some alkaloids isolated recently to 3,4 dihydropyrimidin-2(1H)ones/thiones moiety $[10,11]$. It was first synthesized by Biginelli in 1893 DHPMs as a pot condensation of an aldehyde, diketone, and urea under acidic conditions. These method has low yields, especially in the cases of some substituted aldehydes. To increase the efficiency of the reaction, Biginelli, various catalysts have been used [12].
Biginelli reaction suffers from low yields (20-50\%) of products. Thus, in recent years, several methods to improve the use of $\mathrm{Al}\left(\mathrm{NO}_{3}\right)_{3} \cdot 9 \mathrm{H}_{2} \mathrm{O}$ [13], $\mathrm{ZrCl}_{4}$ [14], zeolites [15], silica sulfuric acid [16], $\mathrm{BF}_{3} \cdot \mathrm{OEt}_{2}$ [17], $\mathrm{CuCl}_{2} \cdot 2 \mathrm{H}_{2} \mathrm{O}$ [18], $\mathrm{SbCl}_{3}$ [19], $\mathrm{RuCl}_{3}$ [20], natural catalyst [21], and glutamic acid [22] have been reported in the literature. However, some of these methods are expensive and harmful to the environment; stoichiometrically, the amount of catalyst, low yields, and incompatibility with other functional groups including product isolation methods is difficult. Therefore, there is still a need for a simple and efficient method for the synthesis of a pot dihydropyrimidinone and thiones under mild conditions. In recent years, eco-friendly industrial application, using green and reusable catalyst, has been studied.

Thus, green chemistry has been defined as a set of principles that reduces or eliminates the use or generation of hazardous chemical materials. It is as part of our current studies on the development of new routes in heterocyclic synthesis [22].

Herein, we want to use the Bismuth (III) sulfate trihydrate as a catalyst in a pot, three-component Biginelli reaction in solvent-free conditions between benzaldehyde, ethyl acetoacetate, and urea for synthesis of DHPMs. This approach 


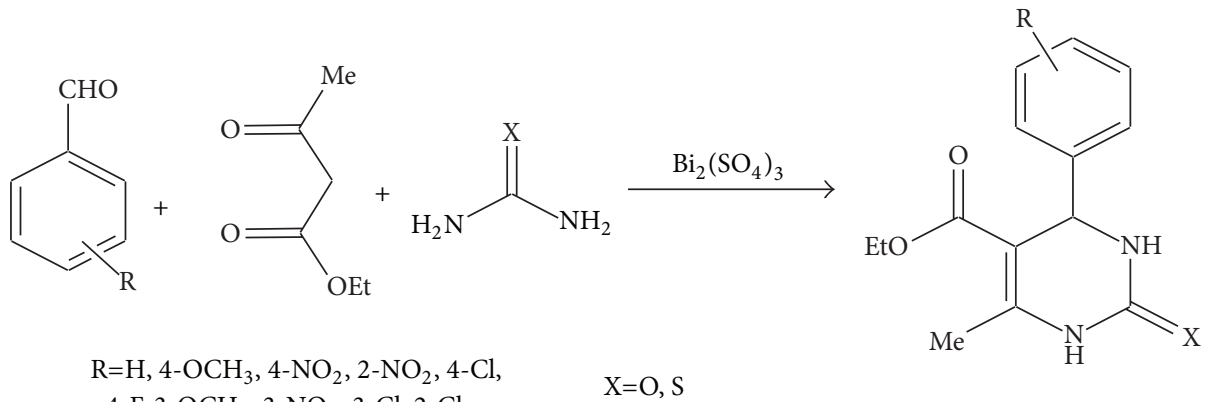

$4-\mathrm{F}, 3-\mathrm{OCH}_{3}, 3-\mathrm{NO}_{2}, 3-\mathrm{Cl}, 2-\mathrm{Cl}$

SCHeme 1: Synthesis of 3,4-dihydropyrimidinones/thiones derivatives.

(Scheme 1) for simple pot Biginelli was a remarkable performance $(>86)$ of dihydropyrimidinones/thiones in a shorter reaction time (60-360 $\mathrm{min}$ ) versus the reaction time required for other catalysts (Scheme 1).

\section{Experimental}

All chemicals were obtained from Merck or Fluka. Melting points were measured on an Electrothermal 9100 apparatus. Silica gel SILG/UV 254 plates were used for TLC. IR spectra were measured on a Shimadzu IR-470 Spectrophotometer. ${ }^{1} \mathrm{H}$ NMR and ${ }^{13} \mathrm{C}$ NMR spectra were determined on Bruker 500 DRX AVANCE instrument at 500 and $125 \mathrm{MHz}$, respectively. The element analyses (C, H, N) were obtained from a Carlo ERBA Model EA 1108 analyzer carried out on Perkin Elmer 240c analyzer.

2.1. General Procedure for the Preparation of 3,4-Dihydropyrimidinones/Thiones $(\mathbf{5} \boldsymbol{a}-\boldsymbol{m})$. A mixture of aldehyde ( $2 \mathrm{mmol})$, ethyl acetoacetate $(2.5 \mathrm{mmol})$, urea/thiourea $(2.5 \mathrm{mmol})$, and Bismuth (III) sulfate trihydrate $(10 \mathrm{~mol} \%)$ was heated with stirring for $60 \mathrm{~min}$ in $90^{\circ} \mathrm{C}$. After cooling, the reaction mixture was poured into crushed ice with stirring. The crude product was filtered, washed with cold water, dried, and recrystallized from 95 ethanol or ethyl acetate to give pure products $(\mathbf{5 a - m})(80-95)$. All compounds were fully characterized by elemental analysis, M.P., IR, CHN, and ${ }^{1} \mathrm{H}$ NMR and ${ }^{13} \mathrm{C}$ NMR spectroscopy. The structures of all synthesized compounds $(\mathbf{5} \mathbf{a}-\mathbf{m})$ have been depicted in Scheme 1.

\subsection{Selected Spectra}

5-(Ethoxycarbonyl)-4-phenyl-6-methyl-3,4-dihydropyrimidin$2(1 \mathrm{H})$-one $(\mathbf{5 a})$. White crystals, m.p. $206-208^{\circ} \mathrm{C}$. IR $(\mathrm{KBr}$, $\left.\mathrm{cm}^{-1}\right): 3245,1725,1635 .{ }^{1} \mathrm{H} \mathrm{NMR}\left(\mathrm{CDCl}_{3} \delta \mathrm{ppm}\right): 1.10(\mathrm{t}$, $\left.3 \mathrm{H}, J=7.2 \mathrm{~Hz}, \mathrm{OCH}_{2} \mathrm{CH}_{3}\right), 2.24\left(\mathrm{~s}, 3 \mathrm{H}, \mathrm{CH}_{3}\right), 3.95(\mathrm{q}, 2 \mathrm{H}$, $\left.J=7.2 \mathrm{~Hz}, \mathrm{OCH}_{2}\right), 5.10(\mathrm{~d}, 1 \mathrm{H}, J=2.2-\mathrm{CH}), 7.28(\mathrm{~m}$, $5 \mathrm{H}, \mathrm{Ar}-\mathrm{H}), 7.77$ (s, 1H, NH), 9.0 (s, 1H, NH). ${ }^{13} \mathrm{C} \mathrm{NMR}$ $\left(\mathrm{CDCl}_{3} \delta \mathrm{ppm}\right): 15.15,19.00,55.22,59.95,101.02,112.22$, $114.15,126.33,126.98,128.45,132.12,149.16,156.77,164.11$.
Anal. Calcd for $\mathrm{C}_{14} \mathrm{H}_{16} \mathrm{~N}_{2} \mathrm{O}_{3}$ (\%): C, 64.62; $\mathrm{H}, 6.15 ; \mathrm{N}, 10.72$. Found: C, 64.58; H, 6.13; N, 10.72.

5-(Ethoxycarbonyl)-4-(4-methoxyphenyl)-6-methyl-3,4-dihydropyrimidin-2(1H)-one (5b). White crystals, m.p. 201-202 ${ }^{\circ} \mathrm{C}$. IR $\left(\mathrm{KBr}, \mathrm{cm}^{-1}\right): 3240,1730,1635 .{ }^{1} \mathrm{H} \mathrm{NMR}\left(\mathrm{CDCl}_{3} \delta \mathrm{ppm}\right)$ : $1.18\left(\mathrm{t}, 3 \mathrm{H}, J=7.15 \mathrm{~Hz}, \mathrm{OCH}_{2} \mathrm{CH}_{3}\right), 2.43\left(\mathrm{~s}, 3 \mathrm{H}, \mathrm{CH}_{3}\right), 3.91$ $\left(\mathrm{s}, 3 \mathrm{H},-\mathrm{O} \mathrm{CH} \mathrm{CH}_{3}\right), 4.12\left(\mathrm{q}, 2 \mathrm{H}, \mathrm{J}=7.15 \mathrm{~Hz}, \mathrm{OCH}_{2} \mathrm{CH}_{3}\right), 5.54$ $(\mathrm{d}, 1 \mathrm{H}, J=2.50-\mathrm{CH}), 6.98(\mathrm{~d}, 2 \mathrm{H}, J=9.10, \mathrm{Ar}-\mathrm{H}), 7.24$ (d, 2H, $J=9.10, \mathrm{Ar}-\mathrm{H}), 7.75$ (s, 1H, NH), 9.33 (s, 1H, NH). ${ }^{13} \mathrm{C} \mathrm{NMR}\left(\mathrm{CDCl}_{3} \delta \mathrm{ppm}\right): 14.55,18.22,56.11,56.40,61.10$, $100.23,116.83,129.38,138.54,147.98,158.09,159.55,165.43$. Anal. Calcd for $\mathrm{C}_{15} \mathrm{H}_{18} \mathrm{~N}_{2} \mathrm{O}_{4}$ (\%): C, 62.06; H, 6.25; N, 9.65. Found: C, 62.04; H, 6.28; N, 9.67.

5-(Ethoxycarbonyl)-4-(4-nitrophenyl)-6-methyl-3,4-dihydropyrimidin-2(1H)-one (5c). White crystals m.p. $211-213^{\circ} \mathrm{C}$. IR $\left(\mathrm{KBr}, \mathrm{cm}^{-1}\right): 3240,1735,1625 .{ }^{1} \mathrm{H} \mathrm{NMR}\left(\mathrm{CDCl}_{3} \delta \mathrm{ppm}\right): 1.14$ $\left(\mathrm{t}, 3 \mathrm{H}, J 7.02 \mathrm{~Hz}, \mathrm{OCH}_{2} \mathrm{CH}_{3}\right), 2.35$ (s, 3H, $\left.\mathrm{CH}_{3}\right), 4.14$ (q, 2H, $\left.J=7.02 \mathrm{~Hz}, \mathrm{OCH}_{2} \mathrm{CH}_{3}\right), 6.09(\mathrm{~d}, 1 \mathrm{H}, J=2.33,-\mathrm{CH}), 7.78$ $(\mathrm{d}, 2 \mathrm{H}, J=8.88, \mathrm{Ar}-\mathrm{H}), 7.89(\mathrm{~s}, 1 \mathrm{H}, \mathrm{NH}), 8.25(\mathrm{~d}, 2 \mathrm{H}$, $J=8.88, \mathrm{Ar}-\mathrm{H}), 9.12(\mathrm{~s}, 1 \mathrm{H}, \mathrm{NH}) .{ }^{13} \mathrm{C} \mathrm{NMR}\left(\mathrm{CDCl}_{3} \delta \mathrm{ppm}\right)$ : $15.02,19.11,56.31,60.75,100.90,120.18,130.77,139.55,154.76$, 155.79, 158.95, 166.44. Anal. Calcd for $\mathrm{C}_{14} \mathrm{H}_{15} \mathrm{~N}_{3} \mathrm{O}_{5}$ (\%): C, 55.06; H, 4.92; N, 13.74. Found: C, 55.12; H, 4.95; N, 13.68.

5-(Ethoxycarbonyl)-4-(4-chlorophenyl)-6-methyl-3,4-dihydropyrimidin-2(1H)-one (5d). White crystals, m.p. $215-216^{\circ} \mathrm{C}$. IR $\left(\mathrm{KBr}, \mathrm{cm}^{-1}\right): 3230,1725,1615 .{ }^{1} \mathrm{H} \operatorname{NMR}\left(\mathrm{CDCl}_{3} \delta \mathrm{ppm}\right): 1.16$ $\left(\mathrm{t}, 3 \mathrm{H}, J=7.09 \mathrm{~Hz}, \mathrm{OCH}_{2} \mathrm{CH}_{3}\right), 2.44\left(\mathrm{~s}, 3 \mathrm{H}, \mathrm{CH}_{3}\right), 4.12(\mathrm{q}$, $\left.2 \mathrm{H}, J=7.09 \mathrm{~Hz}, \mathrm{OCH}_{2} \mathrm{CH}_{3}\right), 5.79(\mathrm{~d}, 1 \mathrm{H}, J=2.27,-\mathrm{CH})$, 7.24 (d, 2H, J = 9.22, Ar-H), 7.79 (s, 1H, NH), 7.84 (d, 2H, $J=9.22, \mathrm{Ar}-\mathrm{H}), 9.20$ (s, $1 \mathrm{H}, \mathrm{NH}) .{ }^{13} \mathrm{C} \mathrm{NMR}\left(\mathrm{CDCl}_{3} \delta \mathrm{ppm}\right)$ : $14.58,18.72$, 56.46, 61.32, 101.92, 119.77, 131.59, 143.57, 154.26, 155.67, 159.98, 165.65. Anal. Calcd for $\mathrm{C}_{14} \mathrm{H}_{15} \mathrm{ClN}_{2} \mathrm{O}_{3}$ (\%): C, 57.12; H, 5.08; N, 9.55. Found: C, 57.08; H, 5.06; N, 9.56.

5-(Ethoxycarbonyl)-4-(3-chlorophenyl)-6-methyl-3,4-dihydropyrimidin-2(1H)-one (5e). White crystals, m.p. 192-193 ${ }^{\circ} \mathrm{C}$. IR $\left(\mathrm{KBr}, \mathrm{cm}^{-1}\right): 3235,1725,1630 .{ }^{1} \mathrm{H} \mathrm{NMR}\left(\mathrm{CDCl}_{3} \delta \mathrm{ppm}\right): 1.11$ $\left(\mathrm{t}, 3 \mathrm{H}, J=7.16 \mathrm{~Hz}, \mathrm{OCH}_{2} \mathrm{CH}_{3}\right), 2.30\left(\mathrm{~s}, 3 \mathrm{H}, \mathrm{CH}_{3}\right), 4.01$ (q, $\left.2 \mathrm{H}, J=7.16 \mathrm{~Hz}, \mathrm{OCH}_{2} \mathrm{CH}_{3}\right), 5.96(\mathrm{~d}, 1 \mathrm{H}, J=2.30,-\mathrm{CH})$, 7.22-7.55 (m, 4H, Ar-H), 7.66 (s, 1H, NH), 9.18 (s, 1H, NH). ${ }^{13} \mathrm{C} \mathrm{NMR}\left(\mathrm{CDCl}_{3} \delta \mathrm{ppm}\right): 14.65,19.04,56.33,60.67,100.89$, $125.31,128.35,128.98,129.83,136.67,143.64,154.78,159.57$, 
165.25. Anal. Calcd for $\mathrm{C}_{14} \mathrm{H}_{15} \mathrm{ClN}_{2} \mathrm{O}_{3}$ (\%): C, 57.14; $\mathrm{H}, 5.10$; N, 9.50. Found: C, 57.13; H, 5.08; N, 9.48.

5-(Ethoxycarbonyl)-4-(4-flurophenyl)-6-methyl-3,4-dihydropyrimidin-2(1H)-one (5f). White crystals, m.p. $182-184^{\circ} \mathrm{C}$. IR $\left(\mathrm{KBr}, \mathrm{cm}^{-1}\right): 3255,1740,1650 .{ }^{1} \mathrm{H} \mathrm{NMR}\left(\mathrm{CDCl}_{3} \delta \mathrm{ppm}\right)$ : $1.18\left(\mathrm{t}, 3 \mathrm{H}, J=7.11 \mathrm{~Hz}, \mathrm{OCH}_{2} \mathrm{CH}_{3}\right), 2.39\left(\mathrm{~s}, 3 \mathrm{H}, \mathrm{CH}_{3}\right), 4.10$ $\left(\mathrm{q}, 2 \mathrm{H}, J=7.11 \mathrm{~Hz}, \mathrm{OCH}_{2} \mathrm{CH}_{3}\right), 6.13(\mathrm{~d}, 1 \mathrm{H}, J=2.20,-\mathrm{CH})$, 7.55 (s, 1H, NH), 7.78 (d, 2H, J = 9.1, Ar-H), 7.95 (d, 2H, $J=9.1, \mathrm{Ar}-\mathrm{H}), 9.21(\mathrm{~s}, 1 \mathrm{H}, \mathrm{NH}) .{ }^{13} \mathrm{C} \mathrm{NMR}\left(\mathrm{CDCl}_{3} \delta \mathrm{ppm}\right)$ : 14.63, 18.98, 56.44, 61.35, 101.89, 122.78, 135.66, 148.08, 155.37, 158.67, 159.65, 165.90. Anal. Calcd for $\mathrm{C}_{14} \mathrm{H}_{15} \mathrm{FN}_{2} \mathrm{O}_{3}$ (\%): C, 60.43; H, 5.39; N, 10.07. Found: C, 60.39; H, 5.36; N, 10.08 .

5-(Ethoxycarbonyl)-4-phenyl-6-methyl-3,4-dihydropyrimidin2(1H)-thione (5g). Yellow crystals, m.p. $208-210^{\circ} \mathrm{C}$. IR $(\mathrm{KBr}$, $\left.\mathrm{cm}^{-1}\right): 3235,1715,1645,1585,1525 .{ }^{1} \mathrm{H}$ NMR $\left(\mathrm{CDCl}_{3} \delta\right.$ ppm): $1.12\left(\mathrm{t}, 3 \mathrm{H}, J=7.25 \mathrm{~Hz}, \mathrm{OCH}_{2} \mathrm{CH}_{3}\right), 2.31(\mathrm{~s}, 3 \mathrm{H}$, $\left.\mathrm{CH}_{3}\right), 4.18\left(\mathrm{q}, 2 \mathrm{H}, J=7.25 \mathrm{~Hz}, \mathrm{OCH}_{2}\right), 5.23(\mathrm{~d}, 1 \mathrm{H}, J=2.15$ -CH), 7.38 (m, 5H, Ar-H), 7.75 (s, 1H, NH), 9.11 (s, 1H, NH). ${ }^{13} \mathrm{C} \mathrm{NMR}\left(\mathrm{CDCl}_{3} \delta \mathrm{ppm}\right): 14.66,18.67,56.87,60.76,100.25$, $112.75,118.39,125.08,128.22,130.14,133.61,153.86,163.42$, 181.48. Anal. Calcd for $\mathrm{C}_{14} \mathrm{H}_{16} \mathrm{~N}_{2} \mathrm{O}_{2} \mathrm{~S}$ (\%): C, 60.85; H, 5.84; N, 10.14; S, 11.60. Found: C, 60.66; H, 5.86; N, 10.14; S, 11.66 .

5-(Ethoxycarbonyl)-4-(3-nitrophenyl)-6-methyl-3,4-dihydropyrimidin-2(1H)-thione (5h). Yellow crystals, m.p. 205$207^{\circ} \mathrm{C}$. IR $\left(\mathrm{KBr}, \mathrm{cm}^{-1}\right): 3260,1740,1635,1580,1545 .{ }^{1} \mathrm{H}$ NMR $\left(\mathrm{CDCl}_{3} \delta \mathrm{ppm}\right): 1.15\left(\mathrm{t}, 3 \mathrm{H}, J=7.12 \mathrm{~Hz}, \mathrm{OCH}_{2} \mathrm{CH}_{3}\right), 2.33$ $\left(\mathrm{s}, 3 \mathrm{H}, \mathrm{CH}_{3}\right), 4.22$ (q, $2 \mathrm{H}, J=7.12 \mathrm{~Hz}, \mathrm{O} \mathrm{CH}_{2} \mathrm{CH}_{3}$ ), 5.75 (d, $1 \mathrm{H}, J=2.11,-\mathrm{CH}), 7.24-7.46(\mathrm{~m}, 4 \mathrm{H}, \mathrm{Ar}-\mathrm{H}), 7.88$ (s, $1 \mathrm{H}$, $\mathrm{NH}), 9.45$ (s, $1 \mathrm{H}, \mathrm{NH}) .{ }^{13} \mathrm{C} \mathrm{NMR}\left(\mathrm{CDCl}_{3} \delta \mathrm{ppm}\right): 14.66$, $19.12,58.12,60.68,101.71,127.45,128.82,129.55,132.39,135.28$, 145.83, 161.02, 165.58, 180.29. Anal. Calcd for $\mathrm{C}_{14} \mathrm{H}_{15} \mathrm{~N}_{3} \mathrm{O}_{4} \mathrm{~S}$ (\%): C, 52.33; H, 4.67; N, 13.08; S, 9.97. Found: C, 52.30; H, 4.66; N, 13.09; S, 9.98 .

5-(Ethoxycarbonyl)-4-(4-methoxyphenyl)-6-methyl-3,4-dihydropyrimidin-2(1H)-thione (5i). Yellow crystals, m.p. 153$155^{\circ} \mathrm{C}$. IR $\left(\mathrm{KBr}, \mathrm{cm}^{-1}\right): 3235,1720,1630,1570,1535 .{ }^{1} \mathrm{H}$ NMR $\left(\mathrm{CDCl}_{3}\right) \delta: 1.18\left(\mathrm{t}, 3 \mathrm{H}, J=7.14 \mathrm{~Hz}, \mathrm{OCH}_{2} \mathrm{CH}_{3}\right), 2.44(\mathrm{~s}, 3 \mathrm{H}$, $\left.\mathrm{CH}_{3}\right), 4.23\left(\mathrm{~s}, 3 \mathrm{H},-\mathrm{O} \mathrm{CH} \mathrm{CH}_{3}\right), 4.38\left(\mathrm{q}, 2 \mathrm{H}, \mathrm{J}=7.14 \mathrm{~Hz}, \mathrm{OCH}_{2}\right.$ $\left.\mathrm{CH}_{3}\right), 5.84(\mathrm{~d}, 1 \mathrm{H}, J=2.22-\mathrm{CH}), 7.31(\mathrm{~d}, 2 \mathrm{H}, J=8.33$, $\mathrm{Ar}-\mathrm{H}), 7.42$ (d, 2H, J = 8.33, Ar-H), 7.56 (s, 1H, NH), 9.21 (s, $1 \mathrm{H}, \mathrm{NH}) .{ }^{13} \mathrm{C} \mathrm{NMR}\left(\mathrm{CDCl}_{3}\right) \delta: 14.87,19.15,56.25,56.49$, $60.78,100.32,115.65,128.86,138.28,145.84,160.35,163.47$, 181.66. Anal. Calcd for $\mathrm{C}_{15} \mathrm{H}_{18} \mathrm{~N}_{2} \mathrm{O}_{3} \mathrm{~S}$ (\%): C, 58.82; $\mathrm{H}, 5.88$; N, 9.15; S, 10.45. Found: C, 58.78; H, 5.86; N, 9.16; S, 10.44 .

5-(Ethoxycarbonyl)-4-(2-chlorophenyl)-6-methyl-3,4-dihydropyrimidin-2(1H)-thione (5j). Yellow crystals, m.p. $218-220^{\circ} \mathrm{C}$. IR $\left(\mathrm{KBr}, \mathrm{cm}^{-1}\right): 3430,3340,1710,1668,1358,1276 .{ }^{1} \mathrm{H}$ NMR $\left(\mathrm{CDCl}_{3} \delta \mathrm{ppm}\right): 1.03\left(\mathrm{t}, J=7.0 \mathrm{~Hz}, 3 \mathrm{H}, \mathrm{CH}_{3}\right), 2.11(\mathrm{~s}$, $\left.3 \mathrm{H}, \mathrm{CH}_{3}\right), 4.20\left(\mathrm{q}, J=7.0,4.28 \mathrm{~Hz}, 2 \mathrm{H}, \mathrm{CH}_{2} \mathrm{O}\right), 5.20(\mathrm{~s}, 1 \mathrm{H}$, $\mathrm{CH}), 6.90-7.25$ (m, 4H, Ar-H), 8.41 (s, 1H, NH), 9.51 (s, 1H, $\mathrm{NH}) .{ }^{13} \mathrm{C} \mathrm{NMR}\left(\mathrm{CDCl}_{3} \delta \mathrm{ppm}\right): 14.52,57.66,61.05,103.27$, $125.55,127.34,128.62,131.44,132.53,144.22,160.11,163.26$, 177.43. Anal. Calcd for $\mathrm{C}_{14} \mathrm{H}_{15} \mathrm{ClN}_{2} \mathrm{O}_{2} \mathrm{~S}$ (\%): C, 54.19; H, 4.84; N, 9.03; S, 10.32. Found: C, 54.14; H, 4.77; N, 9.10; S, 10.25 .
TABLE 1: Bismuth (III) sulfate trihydrate catalyzed synthesis of 5(ethoxycarbonyl)-4-phenyl-6-methyl-3,4-dihydropyrimidin-2(1H)one (5a) in different solvents conditions.

\begin{tabular}{lcccc}
\hline Entry & Solvent & Catalyst (mol\%) & Time (min) & Yield (\%) \\
\hline 1 & Ethanol & 10 & 120 & 25 \\
2 & Methanol & 10 & 120 & 25 \\
3 & Acetonitrile & 10 & 120 & 40 \\
4 & Dichloromethane & 10 & 120 & 33 \\
5 & Chloroform & 10 & 120 & 35 \\
6 & Ethyl acetate & 10 & 120 & 45 \\
7 & THF & 10 & 120 & 20 \\
8 & Solvent-free & 5 & 120 & 65 \\
9 & Solvent-free & 10 & 60 & 89 \\
10 & Solvent-free & 15 & 120 & 78 \\
11 & Solvent-free & 20 & 120 & 55 \\
12 & Solvent-free & 0 & 360 & 10 \\
\hline
\end{tabular}

Reaction conditions: $2 \mathrm{mmol}$ benzaldehyde, $2.5 \mathrm{mmol}$ ethyl acetoacetate, $2.5 \mathrm{mmol}$ urea/thiourea, and Bismuth (III) sulfate trihydrate (10 $\mathrm{mol} \%)$ were refluxed with stirring for $60 \mathrm{~min}$.

TABLE 2: Bismuth (III) sulfate trihydrate catalyzed synthesis of 3,4dihydropyrimidinones/thiones derivatives.

\begin{tabular}{lccccc}
\hline Entry & Compound & Substitution & $\mathrm{X}$ & M.p. $\left({ }^{\circ} \mathrm{C}\right)$ & Yield (\%) \\
\hline 1 & $\mathbf{5 a}$ & $\mathrm{H}$ & $\mathrm{O}$ & $203-204$ & 89 \\
2 & $\mathbf{5 b}$ & 4-Methoxy & $\mathrm{O}$ & $202-203$ & 92 \\
3 & $\mathbf{5 c}$ & 4-Nitro & $\mathrm{O}$ & $212-214$ & 95 \\
4 & $\mathbf{5 d}$ & 4-Chloro & $\mathrm{O}$ & $215-216$ & 93 \\
5 & $\mathbf{5 e}$ & 3-Chloro & $\mathrm{O}$ & $192-193$ & 93 \\
6 & $\mathbf{5 f}$ & 4-Fluro & $\mathrm{O}$ & $182-184$ & 90 \\
7 & $\mathbf{5 g}$ & H & $\mathrm{S}$ & $208-210$ & 88 \\
8 & $\mathbf{5 h}$ & 3-Nitro & $\mathrm{S}$ & $206-208$ & 86 \\
9 & $\mathbf{5 i}$ & 4-Methoxy & $\mathrm{S}$ & $156-158$ & 86 \\
10 & $\mathbf{5 j}$ & 2-Chloro & $\mathrm{S}$ & $218-220$ & 80 \\
11 & $\mathbf{5 k}$ & 2-Nitro & $\mathrm{S}$ & $190-192$ & 81 \\
12 & $\mathbf{5 m}$ & 3-Methoxy & $\mathrm{S}$ & $160-162$ & 85 \\
\hline
\end{tabular}

Reaction conditions: $2 \mathrm{mmol}$ aldehyde, $2.5 \mathrm{mmol}$ ethyl acetoacetate, $2.5 \mathrm{mmol}$ urea/thiourea, and Bismuth (III) sulfate trihydrate (10 mol\%) were refluxed with stirring for $60 \mathrm{~min}$.

5-(Ethoxycarbonyl)-4-(2-nitrophenyl)-6-methyl-3,4-dihydropyrimidin-2(1H)-thione (5k). Yellow crystals, m.p. 190-192 ${ }^{\circ} \mathrm{C}$. IR $\left(\mathrm{KBr}, \mathrm{cm}^{-1}\right): 3338,3289,2996,1685,1572,1355,1310 .{ }^{1} \mathrm{H}$ $\mathrm{NMR}\left(\mathrm{CDCl}_{3} \delta \mathrm{ppm}\right): 1.14\left(\mathrm{t}, J=7.4 \mathrm{~Hz}, 3 \mathrm{H}, \mathrm{CH}_{3}\right), 1.98(\mathrm{~s}$, $\left.3 \mathrm{H}, \mathrm{CH}_{3}\right), 4.15\left(\mathrm{q}, J=7.4,4.55 \mathrm{~Hz}, 2 \mathrm{H}, \mathrm{CH}_{2} \mathrm{O}\right), 5.15(\mathrm{~s}, 1 \mathrm{H}$, $\mathrm{CH}), 6.8-7.38$ (m, 4H, Ar-H): 7.22 (s, 1H, NH), 9.35 (s, 1H, $\mathrm{NH}) .{ }^{13} \mathrm{C} \mathrm{NMR}\left(\mathrm{CDCl}_{3} \delta \mathrm{ppm}\right): 18.37,56.36,60.44,101.48$, $123.21,125.72,126.52,130.26,130.83,142.77,159.61,161.12$, 175.87. Anal. Calcd for $\mathrm{C}_{14} \mathrm{H}_{15} \mathrm{~N}_{3} \mathrm{O}_{4} \mathrm{~S}$ (\%): C, 52.33; H, 4.67; N, 13.08; S, 9.97. Found: C, 52.26; H, 4.65; N, 13.12; S, 9.96.

5-(Ethoxycarbonyl)-4-(3-methoxyphenyl)-6-methyl-3,4-dihydropyrimidin-2(1H)-thione $(\mathbf{5 m})$. Yellow crystals, m.p. 160$162^{\circ} \mathrm{C} . \mathrm{IR}\left(\mathrm{KBr}, \mathrm{cm}^{-1}\right)$ : 3427, 3287, 2967, 1709, 1613, 1300, 1291. ${ }^{1} \mathrm{H} \mathrm{NMR}\left(\mathrm{CDCl}_{3} \delta \mathrm{ppm}\right): 1.22\left(\mathrm{t}, J=7.6 \mathrm{~Hz}, 3 \mathrm{H}, \mathrm{CH}_{3}\right), 2.30$ $\left(\mathrm{s}, 3 \mathrm{H}, \mathrm{CH}_{3}\right), 3.86\left(\mathrm{~s}, 3 \mathrm{H}, \mathrm{Ar}-\mathrm{OCH}_{3}\right), 4.14(\mathrm{q}, J=7.6,4.1 \mathrm{~Hz}$, 
TABLE 3: Biginelli synthesis of 5-ethoxycarbonyl-4-phenyl-6-methyl-3,4-dihydropyrimidin-2(1H)-one: Bismuth (III) sulfate trihydrate in comparison with some recent reports.

\begin{tabular}{|c|c|c|c|c|}
\hline Entry & Conditions & Time (h:min) & Yield (\%) & Reference \\
\hline 1 & $\mathrm{Al}\left(\mathrm{NO}_{3}\right)_{3} \cdot 9 \mathrm{H}_{2} \mathrm{O} / \mathrm{SF}, 80^{\circ} \mathrm{C}$ & $0: 15$ & 98 & [13] \\
\hline 2 & Sulfated tungstate $/ \mathrm{SF}, 80^{\circ} \mathrm{C}$ & $1: 00$ & 92 & [13] \\
\hline 3 & $\mathrm{PPA}-\mathrm{SiO}_{2} / \mathrm{CH}_{3} \mathrm{CN}$, reflux & $1: 00$ & 88 & [13] \\
\hline 4 & $\mathrm{FeCl}_{3}$ immobilized in Al-MCM $41 / \mathrm{CH}_{3} \mathrm{CN}$, reflux & $4: 00$ & 85 & [13] \\
\hline 5 & {$[\mathrm{Hmim}] \mathrm{HSO}_{4} /$ solvent-free, $110^{\circ} \mathrm{C}$} & $0: 20$ & 92 & [13] \\
\hline 6 & Alpha-zirconium sulfophenylphosphonate/SF, $80^{\circ} \mathrm{C}$ & 18:0 & 89 & [13] \\
\hline \multirow[t]{2}{*}{7} & 1, 3-Dichloro-5, 5-dimethylhydantoin $/ \mathrm{CH}_{3} \mathrm{CN}$, reflux & $4: 00$ & 89 & {$[13]$} \\
\hline & $\mathrm{Bi}\left(\mathrm{NO}_{3}\right)_{3}$ & $1: 30$ & 92 & [23] \\
\hline 8 & Bismuth (III) sulfate trihydrate $/ \mathrm{SF}, 90^{\circ} \mathrm{C}$ & $1: 00$ & 89 & In this research \\
\hline
\end{tabular}

$\left.2 \mathrm{H}, \mathrm{CH}_{2} \mathrm{O}\right), 5.18(\mathrm{~s}, 1 \mathrm{H}, \mathrm{CH}), 6.8(\mathrm{~s}, 1 \mathrm{H}, \mathrm{NH}), 6.82-7.84(\mathrm{~m}$, $4 \mathrm{H}, \mathrm{Ar}-\mathrm{H}), 9.42$ (s, $1 \mathrm{H}, \mathrm{NH}) .{ }^{13} \mathrm{C} \mathrm{NMR}\left(\mathrm{CDCl}_{3} \delta \mathrm{ppm}\right)$ : $15.43,19.62,56.35,56.78,61.44,100.02,112.73,126.11,135.56$, 147.67, 161.48, 164.29, 179.37. Anal. Calcd for $\mathrm{C}_{15} \mathrm{H}_{18} \mathrm{~N}_{2} \mathrm{O}_{3} \mathrm{~S}$ (\%): C, 58.82; H, 5.88; N, 9.15; S, 10.45. Found: C, 58.77; H, $5.84 ; \mathrm{N}, 9.16 ; \mathrm{S}, 10.42$.

\section{Results and Discussion}

Bismuth (III) sulfate trihydrate and other salts of Bismuth can be used as a catalyst in the synthesis of organic compounds considered [23-28]. The features of this catalyst could be of high interest because easily separated, environmentally friendly, reusable, clean and affordable. Dihydropyrimidines show a wide range of biological activities. We are interested to develop a simple method for the synthesis of Biginelli reaction DHPMs. Our own study of one-pot three-component Biginelli condensation using Bismuth (III) sulfate trihydrate as a catalyst (Scheme 1) and the reaction with benzaldehyde, ethyl acetoacetate, and urea to afford the product DHPMs as a model reaction (5a) has begun. Synthesis reaction mixture as a model (5a) to determine the appropriate response in the presence of various amounts of Bismuth (III) sulfate trihydrate catalyst under solvent-free conditions (Table 1) was selected. It was found that the yield of compound $\mathbf{5 a}$ was strongly affected by the catalyst amount. The best results under solvent-free conditions (entry 9) in the presence of 10 mol\% catalyst (Table 1 ) were obtained. In various solvents such as ethanol, methanol, acetonitrile, dichloromethane and chloroform, ethyl acetate, THF, and under solvent-free conditions in the presence of $10 \mathrm{~mol} \%$ the catalyst (Table 1) was examined. The best results were obtained in solvent-free conditions at the top and the shortest reaction time (entry 9).

We were successful; 4-dihydropyrimidin-2(1H)-one/thione derivatives of aldehydes and 1,3-dicarbonyl compounds with Bismuth (III) sulfate trihydrate have been synthesized. MCR of benzaldehyde, ethyl acetoacetate, and urea as a model reaction was chosen for optimization. Results are shown in Table 2. With Bismuth (III) sulfate trihydrate as the catalyst, the reaction rate increases dramatically and is easily removed and reused (Table 2). As shown in
Table 1, there is no decrease in catalytic efficiency (entry 12). All reactions were monitored by TLC and forwarded to maximize atom utilization. All compounds with melting points, IR, ${ }^{1} \mathrm{H} \mathrm{NMR},{ }^{13} \mathrm{C} \mathrm{NMR}$, and $\mathrm{CHN}$ techniques were identified. Different aldehydes donating/electron withdrawing for synthesis of DHPMs reacted with excellent yield in available time.

The catalyst was easily recovered by simple filtration after dilution of the reaction mixture with ethyl acetate and was reused after being vacuum dried. $\mathrm{Bi}_{2}\left(\mathrm{SO}_{4}\right)_{3}$ was reused for four runs without significant loss of activity (Run 1: 91\%; Run 2: 89\%; Run 3: 87\%; Run 4: 85\%).

In order to standardize the reaction conditions for the condensation reaction, it was decided to synthesize 3,4dihydropyrimidin-2(1H)-one (5a) from benzaldehyde, urea, and ethyl acetoacetate using Bismuth (III) sulfate trihydrate, and we found that the reaction is fast when compared to other reported methods. The results are compared with the reported methods, and it is clear from Table 3 that the present method is more efficient.

\section{Conclusion}

In conclusion, our Bismuth (III) sulfate trihydrate as a catalyst for the synthesis of dihydropyrimidinones/thiones replaced under solvent-free conditions is displayed. Moderate to good yields of the corresponding DHPMs were obtained. Good yields, One-pot, reused, available time and simple separation experiments under solvent-free conditions with reused catalyst are advantages of this method.

\section{Conflict of Interests}

The author declares no financial conflict of interests.

\section{Acknowledgment}

The author gratefully acknowledges the financial support from the Research Council of Tonekabon Branch, Islamic Azad University. 


\section{References}

[1] T. Yue, M.-X. Wang, D.-X. Wang, G. Masson, and J. Zhu, "Catalytic asymmetric Passerini-type reaction: chiral aluminumorganophosphate-catalyzed enantioselective $\alpha$-addition of isocyanides to aldehydes," Journal of Organic Chemistry, vol. 74, no. 21, pp. 8396-8399, 2009.

[2] B. A. Trofimov, L. V. Andriyankova, K. V. Belyaeva et al., "C2functionalization of 1-substituted imidazoles with aldehydes and electron-deficient acetylenes: a novel three-component reaction," European Journal of Organic Chemistry, no. 9, pp. 1772-1777, 2010.

[3] N. Ma, B. Jiang, G. Zhang, S.-J. Tu, W. Wever, and G. Li, "New multicomponent domino reactions (MDRs) in water: highly chemo-, regio- and stereoselective synthesis of spiro$[1,3]$ dioxanopyridine-4,6-diones and pyrazolo[3,4-b]pyridines," Green Chemistry, vol. 12, no. 8, pp. 1357-1361, 2010.

[4] B. Willy and T. J. J. Müller, "Regioselective three-component synthesis of highly fluorescent 1,3,5-trisubstituted pyrazoles," European Journal of Organic Chemistry, no. 24, pp. 4157-4168, 2008.

[5] M. M. Heravi, B. Baghernejad, H. A. Oskooie, and R. Hekmatshoar, "A novel and facile synthesis of 2-(cyclohexylamino)6,7-dihydro-3-aryl- $1 H$-indole-4(5H)-ones via a one-pot multicomponent reaction," Tetrahedron Letters, vol. 49, no. 42, pp. 6101-6103, 2008.

[6] M. Adib, E. Sheikhi, A. Kavoosi, and H. R. Bijanzadeh, "Synthesis of 2-(alkylamino)-5-alkyl[(2-oxo-2H-chromen-3-yl)carbonyl]amino-3,4-furandicarboxylates using a multi-component reaction in water," Tetrahedron, vol. 66, no. 47, pp. 92639269, 2010.

[7] W.-B. Chen, Z.-J. Wu, Q.-L. Pei, L.-F. Cun, X.-M. Zhang, and W.-C. Yuan, "Highly enantioselective construction of spiro[4H-pyran-3,3/-oxindoles] through a domino knoevenagel/Michael/Cyclization sequence catalyzed by cupreine," Organic Letters, vol. 12, no. 14, pp. 3132-3135, 2010.

[8] S.-L. Wang, F.-Y. Wu, C. Cheng et al., "Multicomponent synthesis of poly-substituted benzo[a]pyrano-[2,3-c]phenazine derivatives under microwave heating," ACS Combinatorial Science, vol. 13, no. 2, pp. 135-139, 2011.

[9] S. R. Kolla and Y. R. Lee, "Efficient one-pot synthesis of $\beta$-phosphono malonates and 2-amino- $4 \mathrm{H}$-chromen-4-ylphosphonate derivatives by ethylenediamine diacetate-catalyzed three-component reactions," Tetrahedron, vol. 68, no. 1, pp. 226237, 2012.

[10] C. O. Kappe, "100 years of the Biginelli dihydropyrimidine synthesis," Tetrahedron, vol. 49, no. 32, pp. 6937-6963, 1993.

[11] C. O. Kappe, "Recent advances in the Biginelli dihydropyrimidine synthesis: new tricks from an old dog," Accounts of Chemical Research, vol. 33, no. 12, pp. 879-888, 2000.

[12] P. Biginelli, "Aldehyde-urea derivatives of aceto- and oxaloacetic acids," Gazzetta Chimica Italiana, vol. 23, pp. 360-413, 1893.

[13] E. Kolvari and M. Mirzaeeyan, " $\mathrm{Al}\left(\mathrm{NO}_{3}\right)_{3} \cdot 9 \mathrm{H}_{2} \mathrm{O}$ : an efficient catalyst for the one-pot synthesis of 3, 4-dihydropyrimidin2(1H)-ones both under reflux or solvent-free conditions," Journal of Chemistry, vol. 2013, Article ID 325268, 5 pages, 2013.

[14] C. V. Reddy, M. Mahesh, P. V. K. Raju, T. R. Babu, and V. V. N. Reddy, "Zirconium(IV) chloride catalyzed one-pot synthesis of 3,4-dihydropyrimidin-2(1H)-ones," Tetrahedron Letters, vol. 43, no. 14, pp. 2657-2659, 2002.
[15] M. G. Kulkarni, S. W. Chavhan, M. P. Shinde et al., "Zeolite catalyzed solvent-free one-pot synthesis of dihydropyrimidin$2(1 \mathrm{H})$-ones: a practical synthesis of monastrol," Beilstein Journal of Organic Chemistry, vol. 5, article 4, 2009.

[16] P. Salehi, M. Dabiri, M. A. Zolfigol, and M. A. Bodaghi Fard, "Silica sulfuric acid: an efficient and reusable catalyst for the one-pot synthesis of 3,4-dihydropyrimidin-2(1H)-ones," Tetrahedron Letters, vol. 44, no. 14, pp. 2889-2891, 2003.

[17] E. H. Hu, D. R. Sidler, and U.-H. Dolling, "Unprecedented catalytic three component one-pot condensation reaction: an efficient synthesis of 5-alkoxycarbonyl-4-aryl-3,4dihydropyrimidin-2(1H)-ones," Journal of Organic Chemistry, vol. 63, no. 10, pp. 3454-3457, 1998.

[18] F. Xu, J.-J. Wang, and Y.-P. Tian, "New procedure for onepot synthesis of 3,4-dihydropyrimidin-2(1H)-ones by Biginelli reaction," Synthetic Communications, vol. 38, no. 8, pp. 12991310, 2008.

[19] I. Cepanec, M. Litvić, M. Filipan-Litvić, and I. Grüngold, "Antimony(III) chloride-catalysed Biginelli reaction: a versatile method for the synthesis of dihydropyrimidinones through a different reaction mechanism," Tetrahedron, vol. 63, no. 48, pp. 11822-11827, 2007.

[20] S. K. De and R. A. Gibbs, "Ruthenium(III) chloride-catalyzed one-pot synthesis of 3,4-dihydropyrimidin-2-(1H)-ones under solvent-free conditions," Synthesis, no. 11, pp. 1748-1750, 2005.

[21] A. M. Elmaghraby, I. A. Mousa, A. A. Harb, and M. Y. Mahgoub, "Three component reaction: an efficient synthesis and reactions of 3, 4-dihydropyrimidin-2(1H)-ones and thiones using new natural catalyst," ISRN Organic Chemistry, vol. 2013, Article ID 706437, 13 pages, 2013.

[22] E. Abbasi and F. Hatamjafari, "Glutamic acid as an efficient catalyst for synthesis of dihydropyrimidinones," Oriental Journal of Chemistry, vol. 29, no. 2, pp. 731-733, 2013.

[23] M. A. Chari, D. Shobha, T. K. Kumar, and P. K. Dubey, "Bismuth (III) nitrate catalyzed one-pot synthesis of 3,4-dihydropyrimidin-2-(1H)-ones: an improved protocol for the Biginelli reaction," Arkivoc, vol. 2005, no. 15, pp. 74-80, 2005.

[24] N. Komatsu, M. Uda, and H. Suzuki, "Bismuth(III) halides and sulfate as highly efficient catalyst for the sulfenylation of carbonyl and related compounds," Synlett, vol. 9, pp. 984-988, 1995.

[25] H. Slimi, Y. Moussaoui, and R. b. Salem, "Synthesis of 3, 4-dihydropyrimidin-2(1H)-ones/thiones via Biginelli reaction promoted by bismuth(III) nitrate or PPh 3 without solvent," Arabian Journal of Chemistry.

[26] K. Ramalinga, P. Vijayalakshmi, and T. N. B. Kaimal, "Bismuth(III)-catalyzed synthesis of dihydropyrimidinones: improved protocol conditions for the Biginelli reaction," Synlett, no. 6, pp. 863-865, 2001.

[27] M. Syamala, "Recent progress in three-component reactions: an update," Organic Preparations and Procedures International, vol. 41, no. 1, pp. 1-68, 2009.

[28] J. M. Bothwell, S. W. Krabbe, and R. S. Mohan, "Applications of bismuth(III) compounds in organic synthesis," Chemical Society Reviews, vol. 40, no. 9, pp. 4649-4707, 2011. 

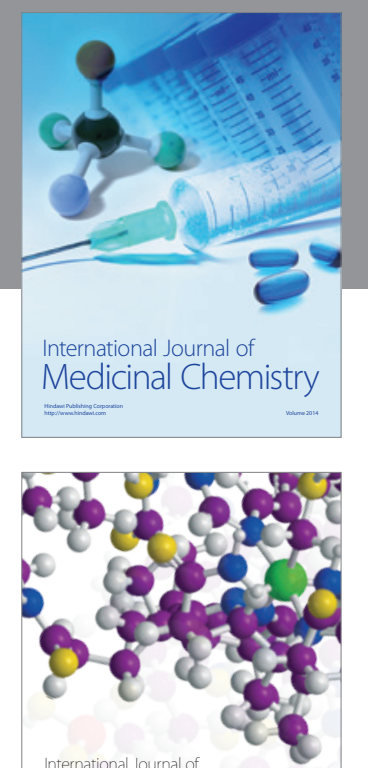

\section{Carbohydrate} Chemistry

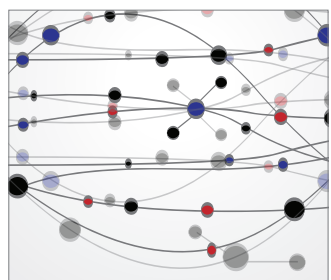

The Scientific World Journal
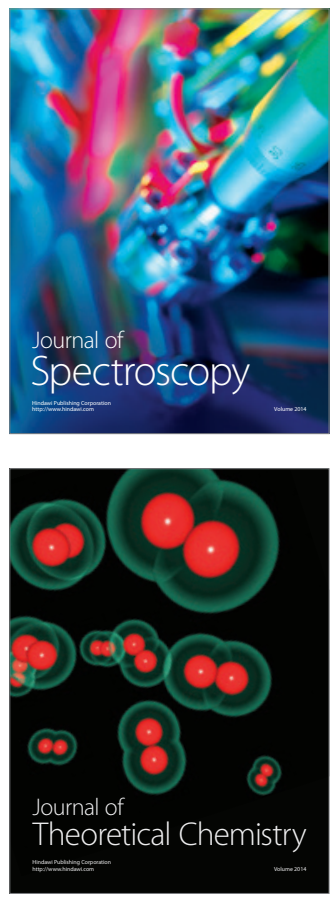
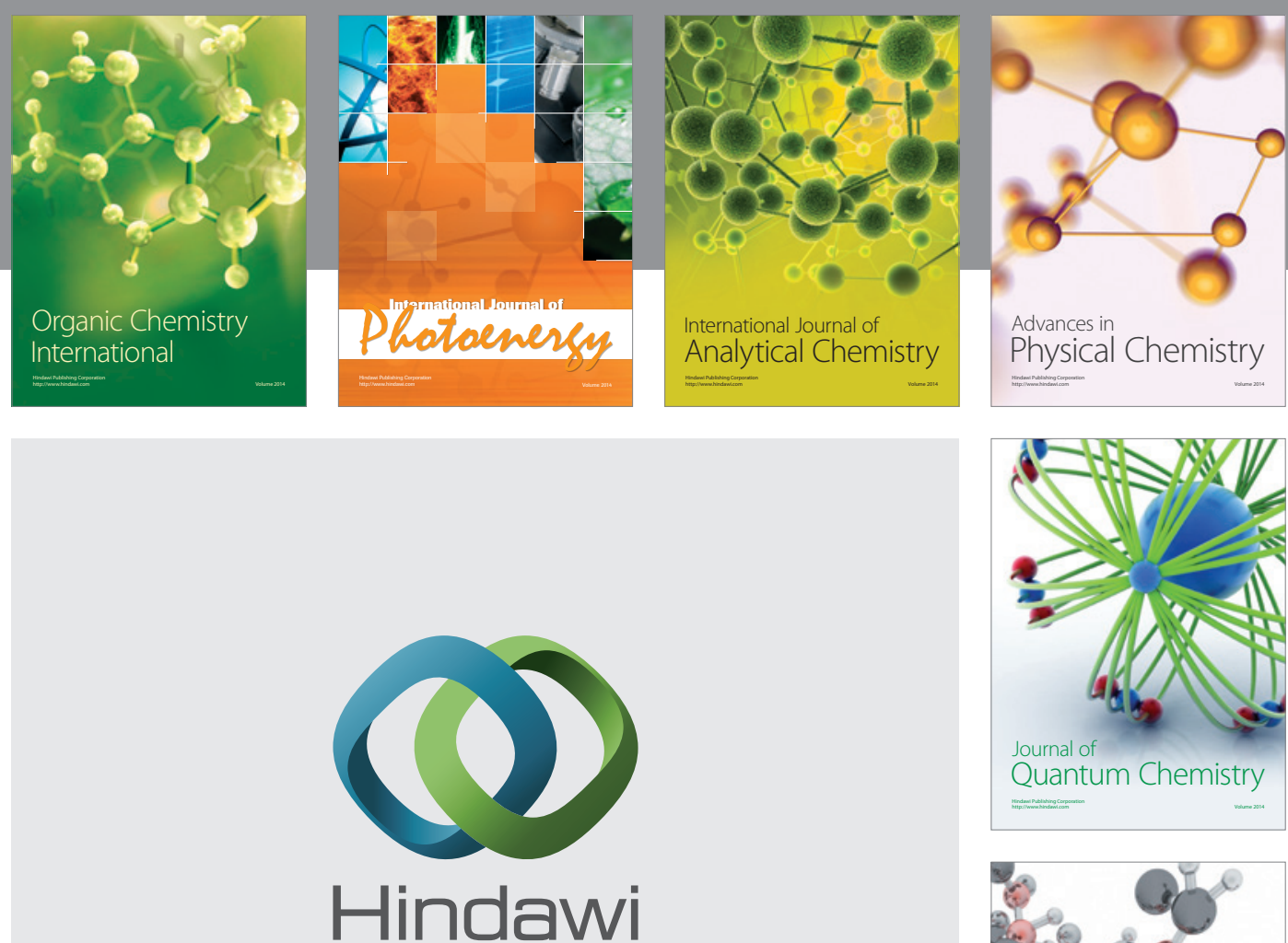

Submit your manuscripts at

http://www.hindawi.com

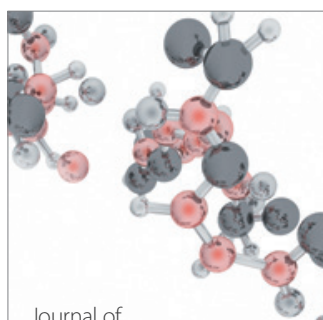

Analytical Methods

in Chemistry

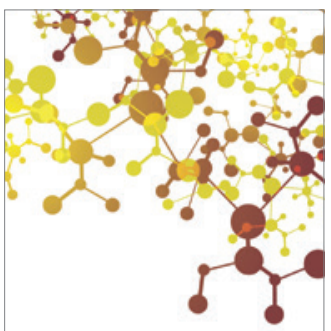

Journal of

Applied Chemistry

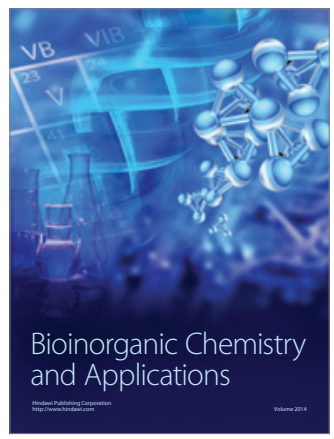

Inorganic Chemistry
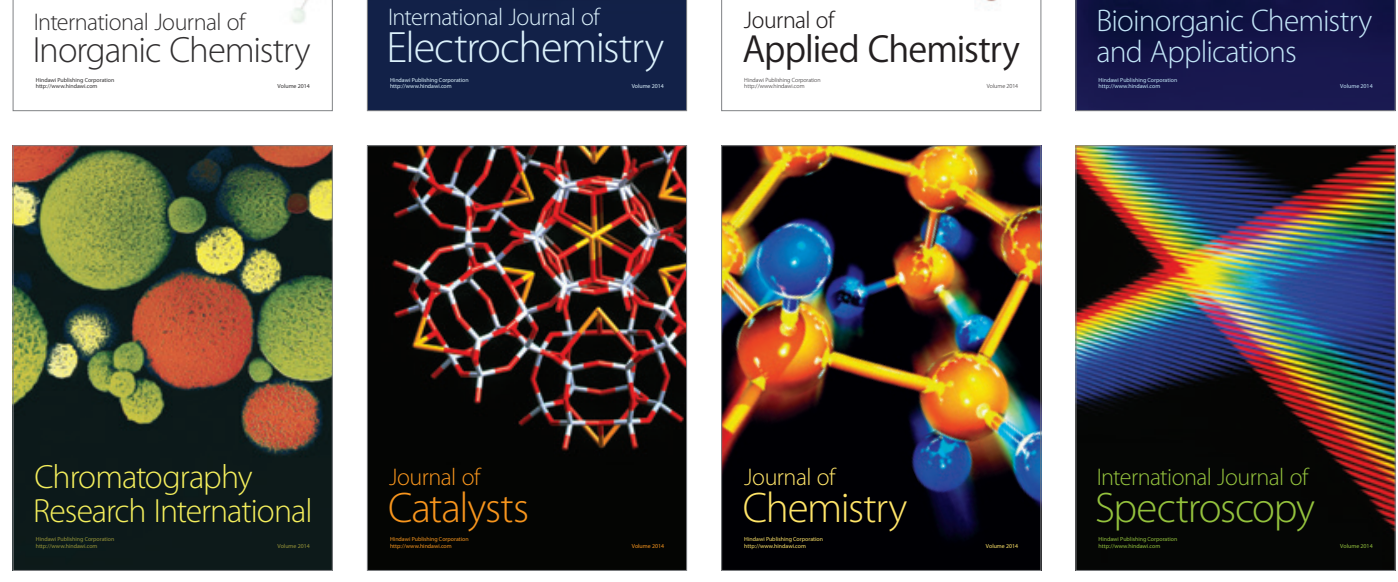